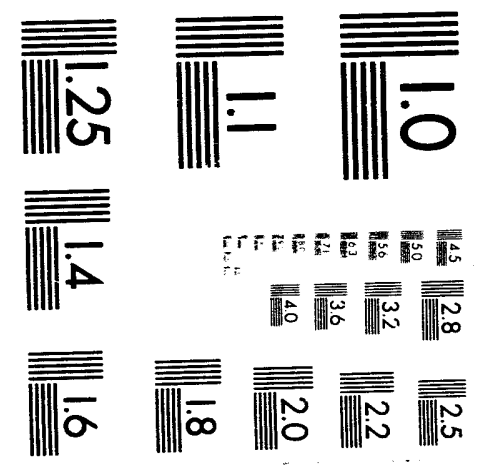



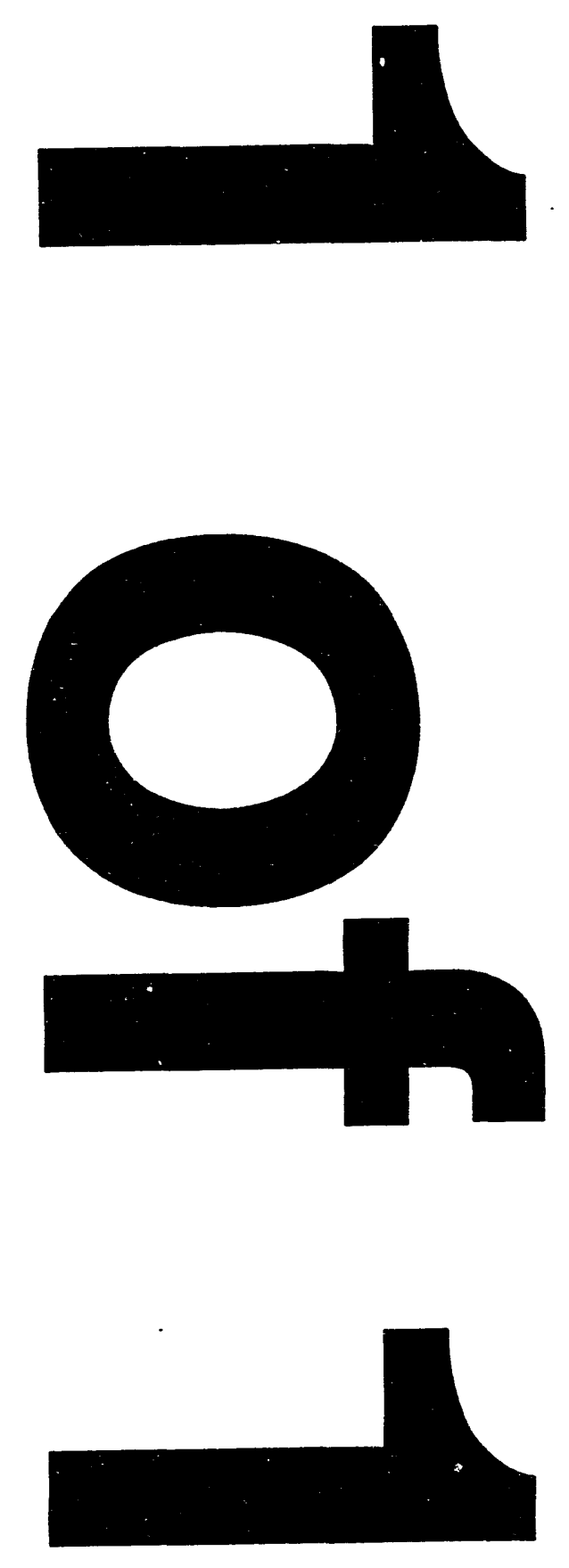


\title{
ANALYSIS AND APPLICATIONS OF QUADRATURE HYBRIDS AS RF CIRCULATORS*
}

\author{
S.M. Hanna and J. Keane \\ National Synchrotron Light Source \\ Brookhaven National Laboratory \\ Upton, NY 11973
}

\section{Abstract}

The operation of a quadrature hybrid as a power combiner is analyzed. The analytical results are compared with data measured experimentally using a $211 \mathrm{MHz}$ cavity. Graphical solutions of the measured cases are in good agreement with analytical predictions. The use of the $90^{\circ}$-hybrid as an RF circulator is also analyzed. The active operation of the harmonic cavity in the NSLS VUV-ring is used to demonstrate this application. This fourth-harmonic cavity is used to change the shape of the bucket potential to lengthen a stored bunch. Thus, a longer stored-beam lifetime can be achieved without compromising the high brightness of the VUV photon beam. If operated actively, the harmonic cavity would present a mismatched load to an RF generator. Thus, a need exists for a circulator. Similarities in operation between the $90^{\circ}$ hybrid and a circulator are discussed.

\section{ANALYTICAL MODEL FOR THE HYBRID AS A RF POWER COMBINER:}

The quadrature hybrid [1], is a four-port network with $90^{\circ}$ phase shift between two of the ports and no phase shift between the other two ports. Refering to Fig. 1, one can write its S-matrix as:

$$
\left(\begin{array}{l}
V_{1}^{-} \\
V_{2}^{-} \\
V_{3}^{-} \\
V_{4}^{-}
\end{array}\right)=\frac{1}{\sqrt{2}}\left(\begin{array}{cccc}
0 & 1 & -j & 0 \\
1 & 0 & 0 & -j \\
-j & 0 & 0 & 1 \\
0 & -j & 1 & 0
\end{array}\right)\left(\begin{array}{c}
V_{1}^{+} \\
V_{2}^{+} \\
V_{3}^{+} \\
V_{4}^{+}
\end{array}\right)
$$

In this section we summerize the results of our analysis for a $90^{\circ}$-hybrid [2]. As shown in Fig.1, the hybrid analysed is wed to combine the power from two transmitters feeding ports 1 and 4. A cavity is being connected to port 2, and a waster lead is connected at port 3.

\section{Assumptions:}

a. The cavity represents an impedance mismatch of $\Gamma_{c}$.

b. The transmitters $T_{A}$ and $T_{B}$ have the same impedance mismatch represented as $\Gamma_{8}$

c. The two transmitters are providing two identical voltages except for a $90^{\circ}$ phase shift.

$$
V_{T_{A}}=V_{8}<0^{\circ} ; V_{T_{2}}=V_{8}<90^{\circ}
$$

*Work performed under the auspices of the U.S. Department of Energy, under contract DE-ACO2-76CH00016.

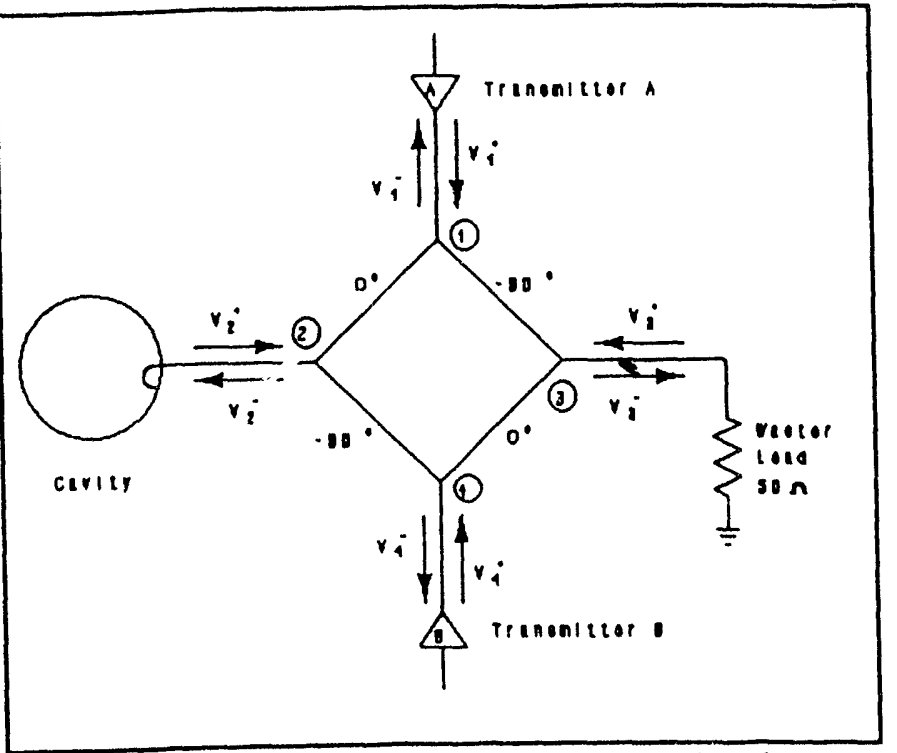

Figure 1. Configuration for the case analyzed.

d. $\quad$ load $\left(Z_{L}=Z_{o}\right.$ of the hybrid) is connected at port 4 .

Approach:

Here we use the linearity of the system and apply the superposition principle. $T_{A}$ is activated while $T_{B}$ is OFF (case 1). Then, $T_{B}$ is activated while $T_{A}$ is OFF (case 2), as follows:

Case 1: Since the active port in this case is port 1 , one needs to solve first for the ratio $V_{1} / V_{1}{ }^{+}$as seen by the transmitter $T_{A}$, when $T_{B}$ is OFF. This is done by using Eq. 1 to solve for $V_{1}{ }^{+}$,as follows:

$$
\left(\begin{array}{l}
V_{1}^{-} \\
V_{2}^{-} \\
V_{3}^{-} \\
V_{4}^{-}
\end{array}\right)=\frac{1}{\sqrt{2}}\left(\begin{array}{cccc}
0 & 1 & -j & 0 \\
1 & 0 & 0 & -j \\
-j & 0 & 0 & 1 \\
0 & -j & 1 & 0
\end{array}\right)\left(\begin{array}{c}
V_{1}^{+} \\
\Gamma_{c} \\
V_{2}^{-} \\
0 \\
\Gamma_{8} V_{4}^{-}
\end{array}\right)
$$

Solving for $\mathrm{V}_{1}^{+}$, one can get ,

$$
V_{1}^{*}=\left(\frac{2+\Gamma_{8} \Gamma_{c}}{2}\right)\left(\frac{1-\Gamma_{8}}{2}\right) V_{s}<0
$$

Now, we can solve for the reflected voltages. This is done by solving Eq. (4).

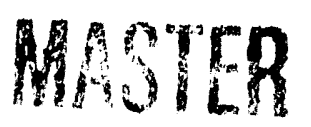

DISTRIBUTION OF THIS DOCUMENT IS UNLIMITED 


$$
\left.\left(\begin{array}{c}
V_{1}^{-} \\
V_{2}^{-} \\
V_{3}^{-} \\
V_{4}^{-}
\end{array}\right)=\frac{1}{\sqrt{2}}\left(\begin{array}{cccc}
0 & 1 & -j & 0 \\
1 & 0 & 0 & -j \\
-j & 0 & 0 & 1 \\
0 & -j & 1 & 0
\end{array}\right)\left(\begin{array}{c}
\frac{2+\Gamma_{c} \Gamma_{c}}{2} \\
\Gamma_{c} V_{2}^{-} \\
0 \\
0 \\
\Gamma_{2} V_{4}^{-}
\end{array}\right) V_{2}<0\right)
$$

giving,

Reflected voltages

\section{Incident voltage}

$$
\begin{aligned}
& V_{1}^{-}=\frac{\Gamma_{c}}{2} \frac{\left(1-\Gamma_{s}\right)}{2} V_{s}<0 \quad V_{1}^{*}=\left(\frac{2+\Gamma_{f} \Gamma_{c}}{2}\right)\left(\frac{1-\Gamma_{s}}{2}\right) V_{s}<0 \\
& V_{2}^{-}=\frac{1}{\sqrt{2}} \frac{1-\Gamma_{s}}{2} V_{s}<0 \quad V_{2}^{+}+\frac{\Gamma_{c}}{\sqrt{2}} \frac{\left(1-\Gamma_{g}\right)}{2} V_{s}<0 \\
& V_{3}^{-}=\frac{i}{\sqrt{2}}\left(1+\Gamma_{s} \Gamma_{2} \frac{\left(1-\Gamma_{s}\right)}{2} V_{s}<0 \quad V_{3}^{*}=0\right. \\
& V_{4}^{-}=-j \frac{\Gamma_{c}}{2}\left(\frac{1-\Gamma_{l}}{2}\right) V_{s}<0 \quad V_{4}^{*}=-j \frac{\Gamma_{g} \Gamma_{c}}{2}\left(\frac{1-\Gamma_{s}}{2}\right) V_{8}<0
\end{aligned}
$$

Case 2: Following the same steps as in Case 1, we solve for $\mathrm{V}_{4}^{+}$, giving

$$
V_{4}^{*}=\left(\frac{2-\Gamma_{8} \Gamma_{c}}{2}\right)\left(\frac{1-\Gamma_{8}}{2}\right) V_{8}<90
$$

Similar to Case 1, we can solve for the incident and reflected voltages at the four ports for this case.

\section{Total Incident and Reflected Voltage:}

By applying the superposition principle, we can get the total voltages at the four ports when both transmitters are ON [2]. The resulting voltages are:

$$
\begin{aligned}
& \text { Transmitter A: } V_{1}^{+}=\left(1+\Gamma_{p} \Gamma_{d} V_{i} ; V_{1}^{-}=\Gamma_{c} V_{1}\right. \\
& \text { Cavity: } V_{2}^{*}=\sqrt{2} \Gamma_{c} V_{l} \quad ; \quad V_{2}^{-}=\sqrt{2} V_{1} \quad(6) \\
& \text { Load: } V_{3}^{*}=0 \quad ; V_{3}^{-}=\sqrt{2} \Gamma_{e} \Gamma_{8} V_{1} \\
& \text { Transmitter } B: V_{4}^{+}=j\left(1-\Gamma_{e} \Gamma_{d}\right) V_{l} \quad ; V_{4}^{-}=j \Gamma_{c} V_{1}
\end{aligned}
$$

where

$$
V_{1}=\frac{\left(1-\Gamma_{2}\right)}{2} V_{8}<0^{\circ}
$$

\begin{tabular}{|c|c|c|c|c|c|c|c|}
\hline $\begin{array}{l}\text { Cav. } \\
\text { Phane }\end{array}$ & $v_{1}^{+}$ & $v_{i}$ & $v_{i}$ & $\mathbf{V}_{2}{ }^{+}$ & $v_{i}$ & $\mathrm{~V}_{4}^{+}$ & $v_{i}$ \\
\hline $0^{\circ}$ & $\begin{array}{l}524 \\
\left(0^{\circ}\right)\end{array}$ & $\begin{array}{l}63 \\
\left(60^{\circ}\right)\end{array}$ & 765 & 100 & 18.7 & 5.48 & 55 \\
\hline $45^{\circ}$ & $\begin{array}{l}990 \\
(.200)\end{array}$ & $\begin{array}{l}530 \\
\left(-1500^{\circ}\right)\end{array}$ & 1142 & 830 & 774 & $\begin{array}{l}900 \\
(1000)\end{array}$ & $\begin{array}{l}485 \\
\text { as? }\end{array}$ \\
\hline $45^{\circ}$ & $\begin{array}{l}1166 \\
\left(0^{\circ}\right)\end{array}$ & $\begin{array}{l}485 \\
\text { (--55") }\end{array}$ & 1116 & 791 & 656 & 354 & $\begin{array}{l}524 \\
1.000\end{array}$ \\
\hline
\end{tabular}

\section{ANALYSIS OF MEASURED DATA}

To verify the above analytical results, measurements were carried out using the configuration shown in Fig. 1. The forward and reflected powers were measured using directional couplers connected to a power meter. The measurement was done using a $211 \mathrm{MHz}$ cavity. The power was measured at three different cavity phase settings $\left(0^{\circ},-45^{\circ}\right.$, and $\left.45^{\circ}\right)$.

The results of the measurements are shown in Table 1 where the voltages are in volts.

Table I

To fully determine the reflection coefficients of the cavity $\left(T_{2}\right)$ and the transmitter $\left(T_{2}\right)$; we use a combination of analytical computation and graphical solutions where we use phasor diagrams and loci for different phasors. To illustrate that we will consider the phasor diagram in Fig. 2 which represents a sample case (cavity at $45^{\circ}$ ).

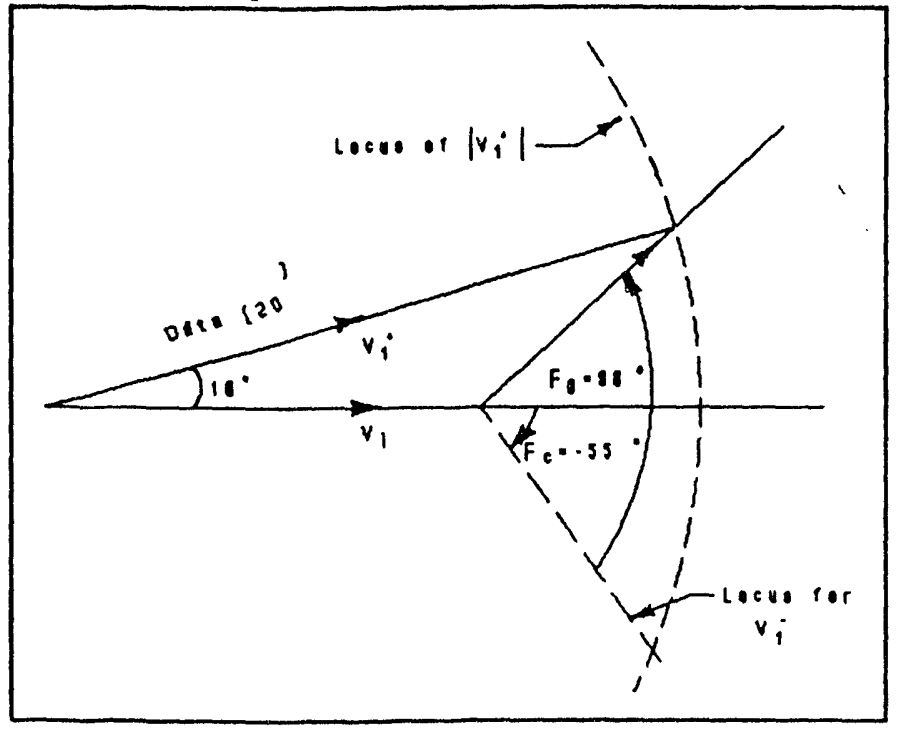

Figure 2. Phasor diagram for graphical solution.

If we consider the phasor $V_{1}$ as the reference, we can find $\phi_{\mathrm{g}}$ (phase angle for $\Gamma_{2}$ ) and $\phi_{\mathrm{c}}$ (phase angle for $\Gamma_{\mathrm{c}}$ ) as shown in Fig 2. Using the data in Table 1, the graphical solution gives

$$
\Gamma_{c}=0.7 \angle-55^{\circ} \text { and } \Gamma_{s}=0.83 \angle 98^{\circ}
$$


Data measured were generally consistant with predictions from the analytical model represented in Eq. (6), except for a systematic phase difference of $10^{\circ}$.

\section{THE $90^{\circ}$-HYBRID AS A CIRCIII,ATOR FOR THE VUV RING HARMONIC CAVITY}

A fourth-harmonic cavity is being used in the VUVstorage ring at the NSLS to change the shape of the bucket potential. The harmonic cavity would present a mismatched load to a generator if operated actively. The need exists for a circulator to steer any power reflected from the harmonic cavity to a waster load. In this section, we demonstrate the use of the $90^{\circ}$-hybrid as circulator. We use the parameters for the harmonic cavity given below as a numerical example [3],.
Main Cavity
Harmonic Cavity
$\mathrm{V}_{\mathrm{MC}}=80 \mathrm{KV}$
$\Psi_{0}=79^{\circ}$
(Rsh = $1 \mathrm{MQ})$

$$
\begin{aligned}
& V_{H C}=20 \mathrm{KV} \\
& \Psi_{H}=-92.8^{\circ}
\end{aligned}
$$$$
(\mathrm{Rsh}=300 \mathrm{kD})
$$

To achieve this goal, we will analyze one of three approaches that we considered [4] for the active operation of the VUV harmonic cavity. In the tuner-compensation approach, the cavity is phased such that the beam current is in quadrature with the cavity gap-voltage. The tuner is operated to compensate for the beam-induced reactive power. The generator current is in-phase with the gap voltage. Lowest generator power requires matched coupling loop to the cavity. Since other practical considerations may require deviations from this ideal case; we have analyzed a general case where coupling can be made different from matched. Referring to Fig 3, we can define the coupling coefficient as

$$
\beta=\frac{R_{1}}{n^{2}(50)}
$$

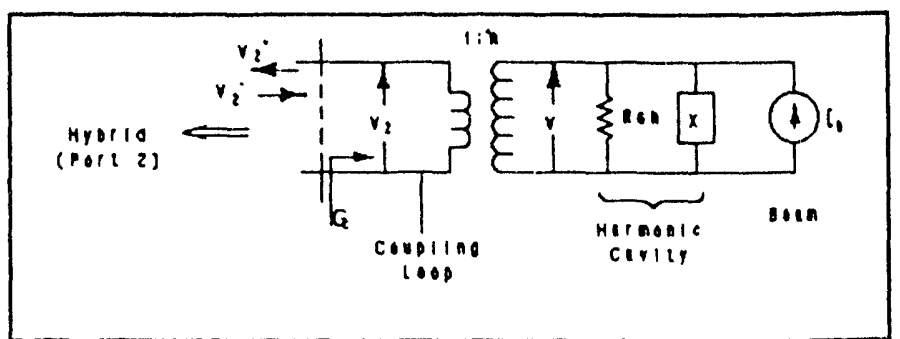

Figure 3. Cavity-port equivalent circuit.

For the conditions discussed above, and analyzed in detail in [4], we can obtain the powers at the four ports as,

Cavity Power: The power dissipatad in the cavity is

$$
P_{w}=\frac{1}{2} \frac{V^{2}}{50 n^{2} \beta}=\frac{1}{2} \frac{V^{2}}{R_{\text {d }}}
$$

Load Power The power dissipated in the waster load is

$$
P_{l}=P_{w}\left[\frac{(\beta-1)^{2}}{4 \beta}\right] \Gamma_{l}^{2}
$$

Transmitter A

$$
P_{A}=P_{w} \frac{\left[(\beta+1)+\Gamma_{\varepsilon}(\beta-1)\right]^{2}-(\beta-1)^{2}}{4 \beta}
$$

\section{Transmitter B}

$$
P_{1}=P_{w} \frac{\left[(\beta+1)-\Gamma_{g}(\beta-1)\right]^{2}-(\beta-1)^{2}}{4 \beta}
$$

\section{Combined Generator Power}

The combined power from transmitter $A$ and transmitter $B$ is

$$
P_{B}=P_{A}+P_{B}=P_{W} \frac{\left[(\beta+1)^{2}-(\beta-1)^{2}\left(1-\Gamma_{B}^{2}\right)\right]}{4 \beta}
$$

From the above equations the power balance gives,

$$
P_{\varepsilon}-P_{l}=P_{w}
$$

From this analysis it can be shown that, under certain conditions (for example the case where the generators can be considered as ideal current sources,$\Gamma_{t}=1$ ), the hybrid's operation is identical to that of a ferrite circulator [4].

\section{Conclusion}

In this report we have analyzed in detail the performance of a $90^{\circ}$-hybrid. The analysis is based on 4-port scattering matrices. Analytical results were compared with experimental measurements. Graphical solutions based on the measured data gave good agreement with our analytical results. The use of the $90^{\circ}$-hybrid as a circulator was demonstrated using the harmonic cavity in the NSLS VUV-ring as an example.

Acknowledgement

We thank M. Thomas and S. Buda for their effort in the measurement of experimental data analyzed in this work.

\section{References}

[1] D.M. Pozar, "Microwave Engineering," Adison-Wesley Publishing Co., NY, p. 411, 1990.

[2] S.M. Hanna and J. Keane, "Analysis of QuadratureHybrids for Accelerator Applications", submitted to Nuc. Inst. \& Meth. in Phys . Research, 1993.

[3] S. Krinsky, et al., "Storage Ring Derelopment at the National Synchrotron Light Source," BNL 46615, 1991.

[4] S.M. Hanna and J. Keane, "Active Operation of the Harmonic Cavity for Bunch Lengthening at the VUVStorage Ring", NSLS Tech. Note \#480, 1993. 


\section{DISCLAIMER}

This report was prepared as an account of work sponsored by an agency of the United States Government. Neither the United States Government nor any agency thereof, nor any of their employees, makes any warranty, express or implied, or assumes any legal liability or responsibility for the accuracy, completeness, or usefulness of any information, apparatus, product, or process disclosed, or represents that its use would not infringe privately owned rights. Reference herein to any specific commercial product, process, or service by trade name, trademark, manufacturer, or otherwise does not necessarily constitute or imply its endorsement, recommendation, or favoring by the United States Government or any agency thereof. The views and opinions of authors expressed herein do not necessarily state or reflect those of the United States Government or any agency thereof. 


\section{1}
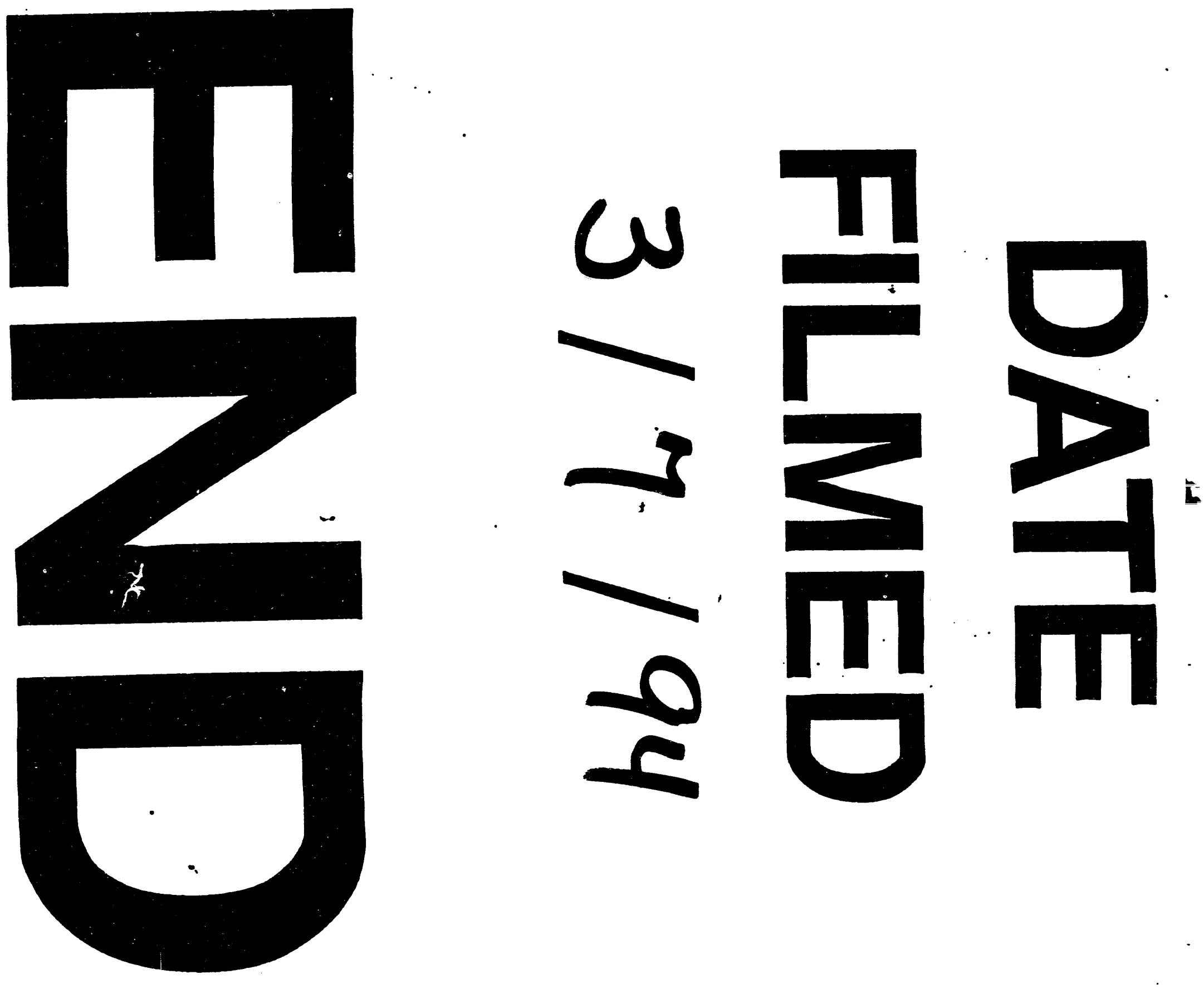
